\title{
Proficiency testing project for brain natriuretic peptide (BNP) and the N-terminal part of the propeptide of BNP (NT-proBNP) immunoassays: the CardioOrmocheck study
}

\author{
Concetta Prontera ${ }^{1}$, Martina Zaninotto ${ }^{3}$, \\ Stefania Giovannini' ${ }^{1}$, Gian Carlo Zucchelli ${ }^{1}$, \\ Alessandro Pilo ${ }^{1}$, Laura Sciacovelli ${ }^{4}$, Mario \\ Plebani $^{3}$ and Aldo Clerico ${ }^{1,2, *}$ \\ ${ }^{1}$ Fondazione Toscana Gabriele Monasterio and CNR \\ Institute of Clinical Physiology, Pisa, Italy \\ ${ }^{2}$ Scuola Superiore Sant Anna, Pisa, Italy \\ ${ }^{3}$ Department of Laboratory Medicine, University- \\ Hospital of Padova, Italy \\ ${ }^{4}$ Biomedical Research Center of Castelfranco \\ Veneto, Italy
}

\begin{abstract}
Background: We organized and conducted a proficiency testing study (CardioOrmocheck) to evaluate the differences in analytical performance of brain natriuretic peptide (BNP) and N-terminal proBNP (NTproBNP) immunoassays.

Methods: Approximately 90 Italian laboratories were involved in the 2005-2007 proficiency testing cycles, while 112 laboratories took part in the 2008 cycle (from January to May 2008). A total of 28 study samples were measured by participating laboratories for a total of 2354 determinations.

Results: The mean total variability for BNP $(50.6 \% \mathrm{CV})$ was significantly higher than that for NT-proBNP $(8.4$ $\% \mathrm{CV})$. In addition, the mean variability due to differences between-methods $(46.4 \% \mathrm{CV})$ comprised the majority of the total variability for BNP. Betweenmethod variability for BNP comprised, on average, $84 \%$ of total variability, while the within-method variability comprised an average of $20.2 \% \mathrm{CV}$. On the contrary, for NT-proBNP the within-method variability (7.3\%CV) represented the majority of total variability (average $75 \%$ ), while between-method variability was smaller $(4.1 \% \mathrm{CV})$. Imprecision around the cut-off value showed marked differences among methods, especially for BNP immunoassay methods. In addition, BNP methods were affected by large systematic differences, for example an average 2.7-fold difference between Access and ADVIA Centaur methods, while agreement between NT-proBNP methods was better (an average 1.2-fold difference between Dimension and ECLIA on the Elecsys methods).
\end{abstract}

*Corresponding author: Prof. Aldo Clerico, MD, Laboratory of Cardiovascular Endocrinology and Cell Biology, Fondazione Toascana G. Monasterio, Via Trieste 41, 56126 Pisa, Italy

Phone: + (39)-0585-493569, Fax: + (39)-0585-493601,

E-mail: clerico@ifc.cnr.it

Received December 17, 2008; accepted March 19, 2009; previously published online April 30, 2009
Conclusions: This multicenter collaborative study demonstrates that there are significant differences in analytical characteristics and measured values among the most popular commercial methods for BNP and NT-proBNP. Clinicians should be very careful when comparing results obtained by laboratories that use different methods.

Clin Chem Lab Med 2009;47:762-8.

Keywords: brain natriuretic peptide (BNP); natriuretic peptides; N-terminal part of the propeptide of BNP (NT-proBNP); quality control.

\section{Introduction}

Cardiac natriuretic peptides, which include atrial natriuretic peptide (ANP), brain natriuretic peptide (BNP) and their related pro-hormones (proANP and proBNP), constitute a complex family of peptide hormones produced and secreted by the heart. ANP and BNP are greatly increased in diseases characterized by an expanded fluid volume, especially heart failure (1). The active peptides, ANP and BNP, are produced by cleavage of the $\mathrm{COOH}$-terminal part of the pro-hormone (proANP and pro-BNP), while the N-terminal fragments of pro-hormone, NT-proANP and NTproBNP, are currently considered inactive $(1,2)$.

Measurement of BNP and its related-peptides is considered a useful marker of myocardial function (1-6), and has been recently included, along with the ECG and radiogram of the thorax, in the first step of the algorithm for diagnosis of heart failure as proposed by the Task Force of the European Society of Cardiology $(7,8)$. Recent meta-analyses $(4,5)$ have confirmed that BNP and NT-proBNP assays have a high degree of diagnostic accuracy and clinical relevance in both acute and chronic heart failure. However, several studies suggest that there are significant differences in the analytical and clinical characteristics, including differences in reference ranges, decision levels and cut-off values, among immunoassays for B-type related peptides that might allow misleading clinical interpretation (9-12).

In order to evaluate the differences in analytical performance and clinical utility of the most commonly used BNP and NT-proBNP immunoassays, a proficiency testing study, based on an external quality assessment scheme called CardioOrmocheck, was implemented in Italy in January 2005. In this article we report the results obtained in this multicenter study following activity from January 2005 to May 2008. 


\section{Materials and methods}

\section{Collaborative study}

The CardioOrmocheck study is a proficiency testing program for the measurement of BNP and NT-proBNP. The study was organized in Italy in 2005 under the patronage of the Study Group of the Cardiovascular Biomarkers of the Italian Society of Clinical Biochemistry (SIBioC) by three accreditated (ISO 9001 accreditation) laboratories: QUALIMED LAB srl, working at CNR Institute of Clinical Physiology and Fondazione G. Monasterio of Pisa, Department of Laboratory Medicine, University-Hospital of Padova, and Biomedical Research Center of Castelfranco Veneto, Italy. These laboratories served in the capacity of central laboratories for the study. On average, 90 Italian laboratories were involved in the testing cycle from 2005 to 2007, while 112 laboratories took part in the 2008 cycle (from January to May 2008). The majority $(84 \%)$ of participating laboratories were Hospital laboratories, while only $16 \%$ were private laboratories.

\section{Sample preparation}

In total, 28 study samples with different BNP and NT-proBNP concentrations were prepared by the three central laboratories (Table 1) according to the ILAC G13 guidelines. These samples were then measured by all participating laboratories for a total of 2354 determinations; some of these same samples were analyzed repeatedly by all laboratories to evaluate within-laboratory variability. A summary of assay methods used to measure BNP and NT-proBNP are shown in Table 2.
Study samples were prepared by pooling several plasma or serum specimens to obtain a sample pool with final volume of about $100 \mathrm{~mL}$ and immediately frozen at $-20^{\circ} \mathrm{C}$. Different sample types were used, including EDTA plasma, Li-heparin plasma, or serum. All samples were tested to ensure absence of HBsAg, anti-HCV, and anti-HIV. Pools were prepared using residual serum or plasma from samples collected from apparently healthy subjects, also divided according to gender, and patients with cardiac diseases, with or without symptomatic heart failure. Demographic information about the 28 study samples are shown in Table 1. Blood samples collected from $\sim 30$ to 50 healthy subjects or patients comprised each study sample. All study participants gave informed consensus for use of their residual blood samples.

Study samples were lyophilized and then sent by mail to all participating laboratories. Lyophilization was performed by polymed (Sambuca, Firenze, Italy) within 2 weeks after preparation of the sample pools. Stored sample pools, were thawed, aliquoted into $\sim 150$ vials of $0.5 \mathrm{~mL}$ each, and then lyophilized. Typical temperature parameters for the lyophilization process were: $-45^{\circ} \mathrm{C}$ for freezing, $-20^{\circ} \mathrm{C}$ for the primary phase, $+25^{\circ} \mathrm{C}$ for secondary phase, and $-60^{\circ} \mathrm{C}$ for condensation. The time for each of the lyophilization steps were $\sim 2 \mathrm{~h}$ for freezing, $24-36 \mathrm{~h}$ for primary phase evaporation, and $\sim 18-24 \mathrm{~h}$ for secondary phase evaporation. The lyophilized materials were reconstituted with $0.5 \mathrm{~mL}$ of distilled water prior to analysis. BNP and NT-proBNP concentrations in all study samples were measured prior to, and following lyophilization, by the central laboratory (CNR Institute of Clinical Physiology in Pisa) in order to evaluate the recovery of BNP and NT-proBNP following the lyophilization

Table 1 Study samples: consensus mean values (mean of the measured values by all methods and laboratories) for BNP and NT-proBNP assays, matrix material, and groups used for blood collection (healthy subjects or cardiac patients with or without symptomatic heart failure).

\begin{tabular}{|c|c|c|c|c|}
\hline $\begin{array}{l}\text { Sample } \\
\text { pool/year }\end{array}$ & $\begin{array}{l}\text { BNP, } \\
n g / L\end{array}$ & $\begin{array}{l}\text { NT-proBNP, } \\
\mathrm{ng} / \mathrm{L}\end{array}$ & Matrix material & Blood collection \\
\hline $1 / 2005$ & 6.2 & 100.3 & Plasma-EDTA & Healthy subjects \\
\hline $2 / 2005$ & 64.8 & 55.7 & Plasma-EDTA & Cardiac patients \\
\hline $3 / 2005$ & 23.7 & 72.5 & Plasma-EDTA & Cardiac patients \\
\hline $4 / 2005$ & 315.8 & 4627 & Plasma-EDTA & Heart failure patients \\
\hline $5 / 2005$ & 38.9 & 865.4 & Plasma-EDTA & Healthy subjects \\
\hline $4 / 2005$ & 202.9 & 4167 & Plasma-EDTA & Heart failure patients \\
\hline $3 / 2005$ & 19.2 & 421.6 & Plasma-EDTA & Healthy subjects \\
\hline $6 / 2005$ & 273.4 & 12,290 & Plasma-EDTA & Heart failure patients \\
\hline $2 / 2006$ & 280.1 & 6620 & Plasma-EDTA & Heart failure patients \\
\hline $5 / 2006$ & 9.3 & 209.7 & Plasma-Li-heparin & Healthy subjects \\
\hline $1 / 2006$ & 51.4 & 817.2 & Plasma-EDTA & Cardiac patients \\
\hline $2 / 2006$ & 295.5 & 6536 & Plasma-EDTA & Heart failure patients \\
\hline $3 / 2006$ & 11.7 & 60.5 & Plasma-EDTA & Healthy subjects \\
\hline $6 / 2006$ & 109.3 & 4886 & Plasma-Li-heparin & Cardiac patients \\
\hline $4 / 2006$ & 30.4 & 896.2 & Plasma-EDTA & Cardiac patients \\
\hline $1 / 2006$ & 48.5 & 781.2 & Plasma-EDTA & Healthy female subjects \\
\hline $4 / 2007$ & 67.3 & 2055 & Plasma-EDTA & Cardiac patients \\
\hline $5 / 2007$ & 10.3 & 528.2 & Plasma-Li-heparin & Healthy subjects \\
\hline $1 / 2007$ & 12.2 & 559.5 & Plasma-EDTA & Healthy subjects \\
\hline $6 / 2007$ & 166.8 & 3763 & Plasma-EDTA & Heart failure patients \\
\hline $7 / 2007$ & 115.2 & 2717 & Plasma-EDTA & Heart failure patients \\
\hline $7 / 2007$ & 133.7 & 2696 & Plasma-EDTA & Heart failure patients \\
\hline $4 / 2007$ & 72.7 & 2157 & Plasma-EDTA & Cardiac patients \\
\hline $2 / 2007$ & 11.0 & 1059 & Serum & Healthy subjects \\
\hline $4 / 2007$ & 78.1 & 2051 & Plasma-EDTA & Cardiac patients \\
\hline $1 / 2008$ & 115.1 & 3272 & Plasma-EDTA & Heart failure patients \\
\hline $3 / 2008$ & 12.7 & 1618 & Plasma-EDTA & Cardiac patients \\
\hline $2 / 2008$ & 25.5 & 1684 & Plasma-EDTA & Cardiac patients \\
\hline
\end{tabular}


Table 2 Listing of BNP and NT-proBNP assay methods used by participant laboratories.

BNP assays

1. SHIONORIA BNP, Shionogi and Co, Ltd, Osaka, Japan (used by $5 \%$ of laboratories).

2. BNP for AxSYM and ARCHITECT platforms, Abbott Diagnostics, Abbott Park, IL, USA (used by $8 \%$ of laboratories).

3. BNP for Access and UniCel Dxl platforms, Beckman, Beckman Coulter, Inc, Fullerton, CA, USA (used by $11 \%$ of laboratories).

4. Triage BNP, Biosite Incorporated, San Diego, CA, USA (used by $15 \%$ of laboratories).

5. Advia Centaur BNP, Siemens Healthcare Diagnostics, Tarrytown, NY, USA (used by $14 \%$ of laboratories). NT-proBNP assays

1. NT-proBNP for Dimension System, Siemens Healthcare Diagnostics, Tarrytown, NY, USA (used by 6\% of laboratories).

2. ECLIA proBNP assay for ELECSYS and Modular platforms, Roche Diagnostics GmbH, Mannheim, Germany (used by $27 \%$ of laboratories).

3. NT-proBNP assay for Immulite 2500 platform, Siemens Medical Solution Diagnostics, Tarrytown, NY, USA (used by $14 \%$ of laboratories).

procedure, as well as the stability of BNP and NT-proBNP in the samples.

\section{Statistical analysis}

Statistical analysis of collected data was performed by the Department of Laboratory Medicine, CNR Institute of Clinical Physiology. Cumulative reports were prepared and sent by mail to each participant laboratory on a periodic basis. Participant laboratories could also access their individual data and cumulative reports online at http://www.ifc.cnr.it/eqas/.

Total variability was estimated by averaging the CVs calculated from the results obtained on each study sample. This variability included both systematic between-method differences and differences between each of the laboratories. The imprecision of the different methods used by participant laboratories was estimated by averaging the CVs of results for the same study sample. Thus, the reported average CVs used for calculation of imprecision profiles was an estimate of the within-method and between-laboratories imprecision that was obtained.

\section{Results}

\section{Study samples}

BNP and NT-proBNP concentrations in all plasma pools were measured in the Laboratory of the CNR Institute of Clinical Physiology before and after lyophilization. Recovery (mean $\pm S E M)$ following lyophilization was significantly higher $(p=0.0006$ by paired t-test) for NT-proBNP $(87.8 \% \pm 4.4 \%)$ measured using the ECLIA method on the Elecsys platform, than for BNP $(66.8 \% \pm 4.3 \%)$ measured using the Beckman ACCESS. Each study sample was measured at least four times using the ECLIA method for NT-proBNP and the ACCESS for BNP. We compared the recovery of BNP for different sample types. Recovery (mean \pm SD) was not significantly different between EDTA plasma $(68.1 \% \pm 24.7 \%)$, heparin plasma $(52.2 \% \pm 13.9 \%)$ or serum $(64.5 \%)$.

Recovery following the lyophilization procedure was also assessed in five EDTA plasma samples with different concentrations of BNP and NT-proBNP and measured by the common methods for these analytes (see Table 3). Peptide concentrations were measured in duplicate using the original EDTA plasma samples and the lyophilized samples following reconstitution. BNP measured with the ADVIA Centaur showed the lowest recovery in lyophilized samples (mean recovery $52 \%$, Table $3 \mathrm{~A}$ ), while NT-proBNP measured using ECLIA on the Elecsys platform the highest recovery (mean recovery $91 \%$, Table $3 \mathrm{~B}$ ). The other BNP and NT-proBNP methods showed similar recoveries, ranging on average from $62 \%$ to $67 \%$. Only the recovery of the ECLIA method was found to be significantly increased compared to the other BNP and NT-proBNP methods assays by means of one way factorial ANOVA $(p<0.001)$. The recovery of the ADVIA method was not significantly different when compared to other

Table 3A Percent recovery following lyophilization in 5 plasma samples measured using the most common BNP immunoassay methods.

\begin{tabular}{|c|c|c|c|c|c|c|c|c|c|c|c|c|}
\hline \multirow[t]{2}{*}{ Sample } & \multicolumn{3}{|c|}{ ACCESS } & \multicolumn{3}{|l|}{ TRIAGE } & \multicolumn{3}{|l|}{ ADVIA } & \multicolumn{3}{|l|}{ AxSYM } \\
\hline & $\begin{array}{l}\text { Before, } \\
\text { ng/L }\end{array}$ & $\begin{array}{l}\text { After, } \\
\text { ng/L }\end{array}$ & $\%$ & $\begin{array}{l}\text { Before, } \\
\text { ng/L }\end{array}$ & $\begin{array}{l}\text { After, } \\
\text { ng/L }\end{array}$ & $\%$ & $\begin{array}{l}\text { Before, } \\
\text { ng/L }\end{array}$ & $\begin{array}{l}\text { After, } \\
\text { ng/L }\end{array}$ & $\%$ & $\begin{array}{l}\text { Before, } \\
\text { ng/L }\end{array}$ & $\begin{array}{l}\text { After, } \\
\text { ng/L }\end{array}$ & $\%$ \\
\hline 1 & 28 & 16 & 57 & 7.6 & 5.7 & 75 & 8.9 & 3.6 & 40 & 27 & 17 & 63 \\
\hline 2 & 51 & 31 & 61 & 14 & 7.8 & 57 & 19 & 8.2 & 43 & 78 & 46 & 59 \\
\hline 3 & 111 & 78 & 70 & 28 & 21 & 77 & 40 & 29 & 73 & 145 & 133 & 92 \\
\hline 4 & 289 & 176 & 61 & 103 & 57 & 55 & 111 & 46 & 41 & 356 & 137 & 39 \\
\hline 5 & 969 & 714 & 74 & 374 & 259 & 69 & 385 & 242 & 63 & 1271 & 732 & 58 \\
\hline
\end{tabular}

Before, assay performed on the original plasma sample prior to lyophilization; After, assay performed on the reconstituted plasma following lyophilization; \%, percent recovery. ACCESS, Access Beckman; TRIAGE, Triage Biosite; ADVIA, ADVIA Centaur Siemens; AxSYM, AxSym Abbott. 
Table 3B Percent recovery following lyophilization in 5 plasma samples measured using the most common NT-proBNP immunoassay methods.

\begin{tabular}{|c|c|c|c|c|c|c|}
\hline \multirow[t]{2}{*}{ Sample } & \multicolumn{3}{|l|}{ ECLIA } & \multicolumn{3}{|c|}{ Dimension } \\
\hline & $\begin{array}{l}\text { Before, } \\
\text { ng/L }\end{array}$ & $\begin{array}{l}\text { After, } \\
\mathrm{ng} / \mathrm{L}\end{array}$ & $\%$ & $\begin{array}{l}\text { Before, } \\
\text { ng/L }\end{array}$ & $\begin{array}{l}\text { After, } \\
\text { ng/L }\end{array}$ & $\%$ \\
\hline 1 & 60 & 57 & 94 & 45.8 & 28 & 62 \\
\hline 2 & 136 & 122 & 90 & 101 & 71 & 71 \\
\hline 3 & 372 & 332 & 89 & 315 & 191 & 61 \\
\hline 4 & 1063 & 951 & 89 & 946 & 586 & 62 \\
\hline 5 & 4499 & 4248 & 94 & 4659 & 2929 & 63 \\
\hline
\end{tabular}

Before, assay performed on the original plasma sample prior to lyophilization; After, assay performed on the reconstituted plasma following lyophilization; \%, percent recovery. ECLIA, ECLIA method with Elecsys platform; Dimension, Dimension Siemens.

BNP methods, as assessed by one way factorial ANOVA, $\mathrm{p}=0.1027$.

\section{Assay methods}

As shown in Table 2, BNP assay methods were more commonly used compared with those for NT-proBNP (53\% vs. $43 \%)$. The ECLIA system for NT-proBNP using the Elecsys and Modular platforms was the most commonly used method, with a utilization ratio of $41 \%$ ( $87 \%$ of utilization ratio among NT-proBNP methods only).

\section{Variability estimation}

The mean total variability (i.e., including variability among all methods and laboratories) for BNP (50.6 $\% \mathrm{CV}$ ) was significantly higher than that for NT-proBNP (8.4\%CV). Furthermore, the mean variability due to differences between-methods $(46.4 \% \mathrm{CV})$ included the predominant component of total variability observed for BNP comprising, on average, $84 \%$ of total variability. The within-method variability showed an average \%CV of 20.2. On the contrary, withinmethod variability for NT-proBNP $(7.3 \% \mathrm{CV})$ represented the greater part of total variability, an average of $75 \%$, while the between-method variability was smaller showing a \%CV of 4.1.

The imprecision profiles for the most common BNP assays in the survey are shown in Figure 1A. Large variability in imprecision among these methods was found. Imprecision profiles for the most common NTproBNP methods are given in Figure 1B. The ECLIA method on the Elecsys and Modular platforms showed better performance than the Dimension method, with a CV of $<10 \%$ for the majority of the working range of the assay.

The BNP methods showed good correlation with correlation coefficient values ranging from $R=0.907$ (correlation between ACCESS Beckman and ADVIA Siemens) to 0.986 (correlation between ACCESS Beckman and TRIAGE Biosite). However, a significant difference was found between mean BNP values measured with the Siemens ADVIA Centaur method and those measured using the other three methods as detected using the Scheffé test after ANOVA using log-transformed data (see Figure 2A). In particular, the ADVIA method showed marked lower values compared with the AxSYM. An average difference of $-20.8 \%$, calculated as ADVIA-AxSYM/mean value \% ( $p<0.0001$ by Wilcoxon signed rank test), while the ACCESS showed an average difference of $-24.9 \%$, calculated as ADVIA-ACCCES/mean value \% $(p<0.0001)$. In addition, the AxSYM method gave BNP values significantly lower than those measured with the ACCESS method; on average a difference of
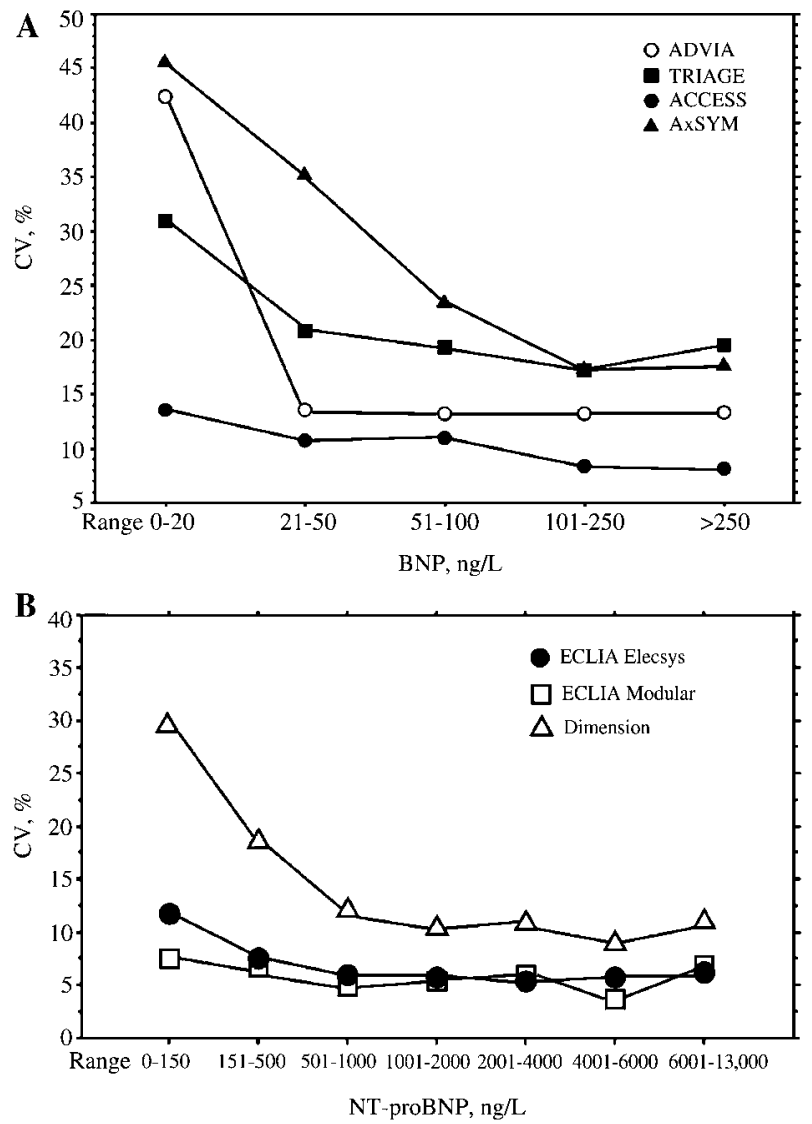

Figure 1 Imprecision profiles of BNP (Part A) and NTproBNP (Part B) immunoassays most commonly used in the survey.

$\mathrm{CV}$ values represent the mean between-laboratories variability for each method. Samples were grouped according to concentration ranges (5 for BNP and 7 for NT-proBNP, respectively) in order to obtain more accurate estimates of the mean $\mathrm{CV}$ values. 
A

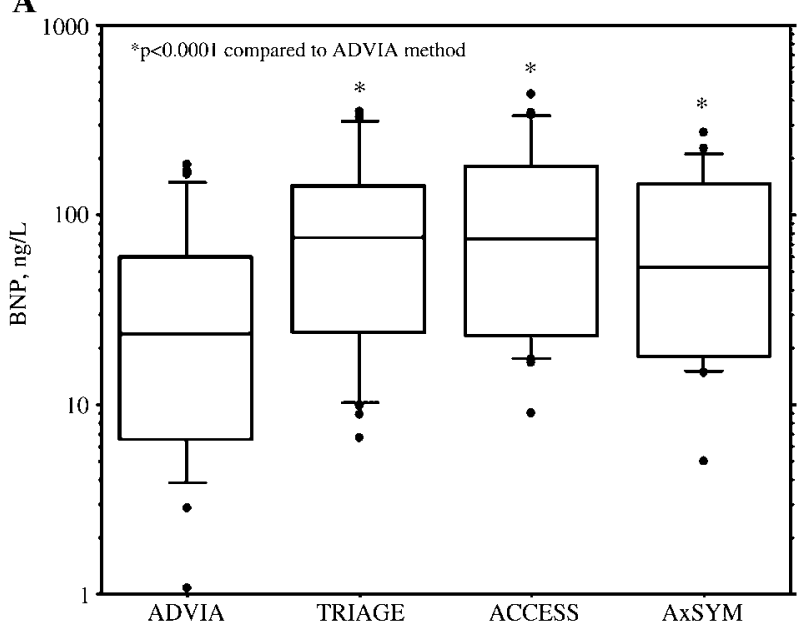

B

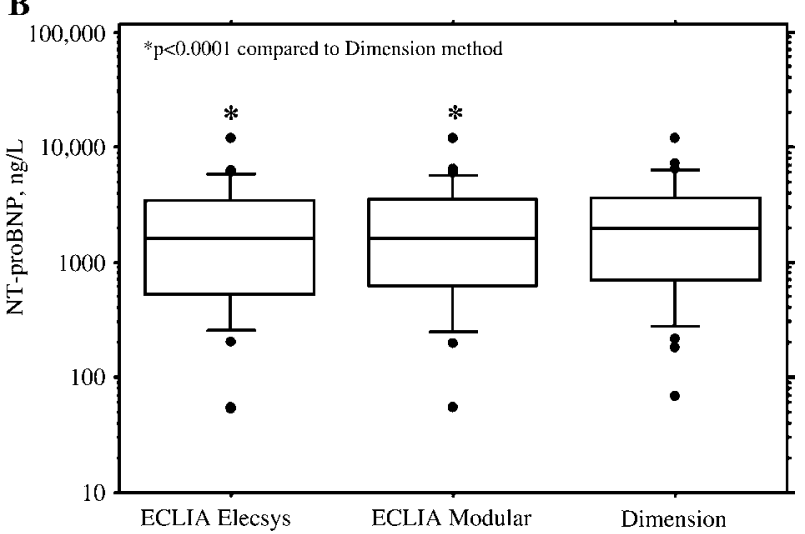

Figure 2 Box (distribution) plot of BNP (Part A) and NTproBNP (Part B) values measured by the most common methods.

Data are reported as boxes indicating the 10th, 25th, 50th (median), 75th and 90th percentiles of BNP and NT-proBNP values measured in the 28 study samples; the outliers are indicated as separated black circles. The asterisk $(*)$ indicates the level of statistical significance $(p<0.0001)$ compared to the ADVIA Centuar method, for BNP methods, and to the Dimension method, for NT-proBNP methods, respectively.

$-8.4 \%$ calculated as AxSYM-ACCCES/mean value \% $(p<0.0005)$. Finally, a significant difference was found between the mean value measured with the two ECLIA and Dimension methods (Figure 2B), although the difference was smaller than that seen for BNP methods.

\section{Evaluation of matrix effects}

In order to evaluate possible matrix effects on BNP and NT-proBNP immunoassays, some study samples with different matrix materials were prepared and assayed by all participant laboratories. These included one serum sample and three heparinized plasma samples (Table 1). The effect of sample matrix on the total variability of BNP or NT-proBNP assays was assessed by means of a stepwise multiple regression analysis. This analysis included total variability as the dependent variable and the concentration of BNP or NT-proBNP (log-transformation of original data) and matrix material (expressed as a dummy variable with
1 for EDTA and 2 for serum or heparin plasma) as independent variables. The matrix material variable showed no significant independent effect on the linear negative regression between total variability and BNP $(R=0.445, p=0.0137)$ or NT-proBNP $(R=0.378$, $\mathrm{p}=0.0395)$.

\section{Discussion}

\section{Theoretical limitations and aims of the study}

There are some theoretical limitations concerning a proficiency testing study for BNP and NT-proBNP immunoassay methods. The specifications for measurement of BNP recommend the use of EDTA plasma samples, while serum is the preferred sample for NTproBNP (10). Furthermore, the use of EDTA plasma samples is not recommended by the manufacturers for some NT-proBNP immunoassays, such as the method used on the Dimension platform (13). Following the manufacturer's recommendations and quality specifications, at least two different human blood samples should be used in a proficiency testing study for BNP and NT-proBNP: EDTA plasma for BNP methods, and serum for NT-proBNP. Alternatively, an artificial material could be prepared, which is commutable for all BNP and NT-proBNP immunoassay methods. Unfortunately, to our knowledge, this type of material is not presently available.

In order to reduce the in vitro degradation, especially for BNP, and to make the distribution and measurement of blood samples easier, we decided to use lyophilized plasma and serum samples only. It is important to note that both plasma or serum samples are actually used for the measurement of BNP and its related peptides. Thus, our protocol is in accordance with actual laboratory practice.

The primary aim of the CardioOrmocheck study is to answer the theoretical limitations of a proficiency study of methods for BNP and NT-proBNP that use human plasma or serum samples. Although some differences in the recovery of lyophilized samples were observed between immunoassay methods (Table 3), the results obtained in the present study are in agreement with previously reported data $(2,14)$. This supports the suitability of this material for between-method comparisons. The results of the CardioOrmocheck study suggest that it is possible to perform a reliable proficiency testing study for BNP and NT-proBNP immunoassay methods using lyophilized serum or plasma samples pools collected from well-characterized groups of healthy subjects or cardiac patients with or without heart failure.

Another important aim of the CardioOrmocheck study was to mimic the actual laboratory and clinical situations associated with measurement of BNP and NT-proBNP. Although the results of the CardioOrmocheck study are relevant to conditions seen in Italian laboratories, some of the results of this study may have a more general relevance, as discussed above. 
Results and discussion

Since BNP and NT-proBNP differ in biochemical structure, molecular weight, biological activity and degradation pathways $(1,2,12)$, it is not surprising that BNP and NT-proBNP assay methods may also have different analytical characteristics and quality specifications. The biologically active peptide, BNP, shows a shorter half-life and a more rapid degradation rate both in vivo and in vitro than the inactive peptide, NTproBNP $(1,2)$. As a result, the biological variation of NT-proBNP is smaller than that of BNP (15). Furthermore, BNP degradation in vitro is minimized by the use of EDTA and/or other inhibitors of plasma proteases, such as the aprotinin (2, 9-12). Indeed, quality specifications for BNP assays recommend the use of EDTA plasma samples $(10,11)$. On the contrary, previous data (16-18), as well as the results of the present study, suggest that the NT-proBNP assay can be performed in serum as well as EDTA or heparinized plasma. However, possible matrix effects due to the use of anticoagulation with EDTA or heparin should be tested $(12,16-18)$. Data from this study demonstrate that there are wide differences in analytical characteristics among immunoassay methods. In particular, the imprecision around the cut-off values (corresponding to $50-100 \mathrm{ng} / \mathrm{L}$ for BNP and $100-150 \mathrm{ng} / \mathrm{L}$ for NT-proBNP, respectively) $(4,12)$ showed marked differences among methods (Figure 2). Only the two ECLIA methods for NT-proBNP showed imprecision profiles $\leq 10 \% \mathrm{CV}$ around the cut-off values (i.e., about 100-150 ng/L), while the Dimension method for NT-proBNP and Access and ADVIA Siemens for BNP (cut-off value about $50 \mathrm{ng} / \mathrm{L}$ ) showed imprecision below $20 \%$. The other immunoassays demonstrated even worse imprecision values.

The results of the CardioOrmocheck study confirm that the most common BNP immunoassays are affected by large systematic differences (on average, 2.7fold between the Access and ADVIA Centaur methods, Figure 2A). Agreement between NT-proBNP methods was better (on average 1.2-fold difference between the Dimension and ECLIA on Elecsys platform methods, Figure $2 \mathrm{~B}$ ). These results were largely expected because all the commercial NT-proBNP methods tested in the study, the two ECLIA methods and the Dimension method, use antibodies and standard materials from the same source (Roche Diagnostics), while BNP methods use different antibodies and standard materials (2, 9-12). In particular, the automated BNP immunoassays on the AxSYM and ACCESS, and the POCT method on the TRIAGE Biosite shared similar BNP values (Figure 2A). Only the fully-automated ADVIA Centaur method and the immunometric assay (IRMA) by Shionogi (data not shown), which use the same antibodies, showed significantly lower results compared with the other methods. The present study is in close agreement with previous studies comparing the analytical performance and diagnostic accuracy of some BNP assays (14). The results of CardioOrmocheck study confirm that is not reasonable to suggest similar cutoff or decision values for all BNP assays $(2,12,14)$.

\section{Conclusions}

The present study suggests that there are several theoretical and practical limitations to performing a proficiency study for BNP and NT-proBNP immunoassay methods due to non-homogeneity and possible noncommutability of human plasma and serum samples. In addition, there are differences in recovery of analyte following lyophilization. Moreover, our data confirm that there are marked differences in the analytical characteristics and measured values among the most commonly used commercial methods for BNP and NT-proBNP. Therefore, clinicians should carefully evaluate results obtained by different laboratories, especially when different methods are used. Furthermore, our findings confirm that standardization is necessary for immunoassay methods, especially for those that measure BNP.

\section{References}

1. Clerico A, Recchia FA, Passino C, Emdin M. Cardiac endocrine function is an essential component of the homeostatic regulation network: physiological and clinical implications. Am J Physiol Heart Circ Physiol 2006;290: H17-29.

2. Clerico A, Emdin M. Diagnostic accuracy and prognostic relevance of the measurement of the cardiac natriuretic peptides: a review. Clin Chem 2004;50:33-50.

3. Doust JA, Glasziou PP, Pietrzak E, Dobson AJ. A systematic review of the diagnostic accuracy of natriuretic peptides for heart failure. Arch Intern Med 2004;164:1978-84.

4. Clerico A, Fontana M, Zyw L, Passino C, Emdin M. Comparison of the diagnostic accuracy of brain natriuretic peptide (BNP) and the N-terminal part of the propeptide of BNP immunoassays in chronic and acute heart failure: a systematic review. Clin Chem 2007;53:813-22.

5. Ewald B, Ewald D, Thakkinstian A, Attia J. Meta-analysis of $B$ type natriuretic peptide and $\mathrm{N}$-terminal pro $\mathrm{B}$ natriuretic peptide in the diagnosis of clinical heart failure and population screening for left ventricular systolic dysfunction. Intern Med J 2008;38:101-13.

6. Tang WH, Francis GS, Morrow DA, Newby LK, Cannon CP, Jesse RL, et al. National Academy of Clinical Biochemistry Laboratory Medicine practice guidelines: clinical utilization of cardiac biomarker testing in heart failure. Circulation 2007;116:e99-109.

7. Nieminen MS, Bohm M, Cowie MR, Drexler H, Filippatos GS, Jondeau G, et al. Executive summary of the guidelines on the diagnosis and treatment of acute heart failure: the Task Force on Acute Heart Failure of the European Society of Cardiology. Eur Heart J 2005;26:384-416.

8. Swedberg K, Cleland J, Dargie H, Drexler H, Follath F, Komajda M, et al. Guidelines for the diagnosis and treatment of chronic heart failure: executive summary (update 2005): The Task Force for the Diagnosis and Treatment of Chronic Heart Failure of the European Society of Cardiology. Eur Heart J 2005;26:1115-40.

9. Clerico A, Del Ry S, Giannessi D. Measurement of natriuretic cardiac hormones (ANP, BNP, and related peptides) 
in clinical practice: the need for a new generation of immunoassay methods. Clin Chem 2000;46:1529-34.

10. Apple FS, Panteghini M, Ravkilde J, Mair J, Wu AHB, Tate J, et al. On behalf of the committee on standardization of markers of cardiac damage of the IFCC. Quality specifications for B-type natriuretic peptide assays. Clin Chem 2005;51:486-93.

11. Apple FS, Wu AH, Jaffe AS, Panteghini M, Christenson $\mathrm{RH}$, Cannon CP, et al. National Academy of Clinical Biochemistry and IFCC Committee for Standardization of Markers of Cardiac Damage Laboratory Medicine practice guidelines: analytical issues for biomarkers of heart failure. Circulation 2007;116:e95-8.

12. Vittorini S, Prontera T, Zucchelli GC, Clerico A. Cardiac natriuretic hormones: methodological aspects. ImmunoAnalyse Biol Spec 2007;22:209-72.

13. Siemens Technical Bulletin. Dimension clinical chemistry system: heterogeneous immunoassay module. REF.RF423A, 2008.

14. Clerico A, Prontera C, Emdin M, Passino C, Storti S, Poletti $R$, et al. Analytical performance and diagnostic accuracy of immunometricassays for the measurement of plasma BNP and NT-proBNP concentrations. Clin Chem 2005;51:445-7.

15. Clerico A, Carlo Zucchelli G, Pilo A, Passino C, Emdin M. Clinical relevance of biological variation: the lesson of brain natriuretic peptide (BNP) and NT-proBNP assay. Clin Chem Lab Med 2006;44:366-78.

16. Collinson PO, Barnes SC, Gaze DC, Galasko G, Lahiri A, Senior R. Analytical performance of the $\mathrm{N}$-terminal pro B type natriuretic peptide (NT-proBNP) assay on the Elecsys 1010 and 2010 analysers. Eur J Heart Fail 2004; 15:365-8.

17. Jarausch J, Garcia-Beltran L, Gremmelr B, Hensel-Wiegel K, Luthe $\mathrm{H}$, Meisel S, et al. Multicentre evaluation of the second generation Elecsys NT-proBNP assay. (Abstract B-137) Clin Chem 2008;54(Suppl 6):A93.

18. Prontera C, Zucchelli GC, Vittorini S, Storti S, Emdin M, Clerico A. Comparison between analytical performances of polyclonal and monoclonal electrochemiluminescence immunoassays for NT-proBNP. Clin Chim Acta 2009;400:70-3. 